\title{
Color transformation-based dynamic voltage scaling for mobile AMOLED displays
}

\author{
Jun-Hyeok Park, Zenebe Maregn, and Young-Jin Kim ${ }^{\text {a) }}$ \\ Department of Electrical and Computer Engineering, Ajou University, \\ San 5, Woncheon-dong, Yeongtong-gu, Suwon 443-749, Republic of Korea \\ a)youngkim@ajou.ac.kr
}

\begin{abstract}
AMOLED displays have been emerged as a new technology recently but have a significant portion of the total power consumption in mobile devices. In this paper, we propose a novel approach which combines color transformation and dynamic voltage scaling (DVS) synergistically in the aspects of both power saving and visual quality. It adaptively selects an allowable perceptuality and saturation threshold based on the luminance value of an input image, then transforms the colors of image pixels and performs DVS while satisfying human visuality. To implement it, a board interfaced with a mobile AMOLED display module is developed. Experiments show that the proposed approach achieves on average $29.6 \%$ power saving for still images with high satisfaction to human visuality.
\end{abstract}

Keywords: AMOLED, dynamic voltage scaling, color transformation, mobile low-power display

Classification: Electronic displays

\section{References}

[1] C.-C. Lai and C.-C. Tsai: IEEE Trans. Consum. Electron. 54 (2008) 669. DOI:10.1109/TCE.2008.4560145

[2] A. Bartolini, M. Ruggiero and L. Benini: EMSOFT (2009) 21. DOI:10.1145/ 1629335.1629339

[3] M. Stewart, R. S. Howell, L. Pires and M. K. Hatalis: IEEE Trans. Electron Devices 48 (2001) 845. DOI:10.1109/16.918227

[4] A. Nathan, G. R. Chaji and S. J. Ashtiani: J. Disp. Technol. 1 (2005) 267. DOI:10.1109/JDT.2005.858913

[5] J. Wang, X. Lin and C. North: Technical Report TR-12-09, Department of Computer Science, Virginia Tech (2012).

[6] M. Dong and L. Zhong: MobiSys (2011) 85. DOI:10.1145/1999995.2000004

[7] K. W. Tan, T. Okoshi, A. Misra and R. K. Balan: UbiComp (2013) 573. DOI: $10.1145 / 2493432.2493445$

[8] B. Anand, K. Thirugnanam, J. Sebastian, P. G. Kannan, A. L. Ananda, M. C. Chan and R. K. Balan: MobiSys (2011) 57. DOI:10.1145/1999995.2000002

[9] B. Anand, K. Li and A. L. Ananda: ICMU (2014) 21. DOI:10.1109/ICMU. 2014.6799052

[10] D. Shin, Y. Kim, N. Chang and M. Pedram: DAC (2011) 53. DOI:10.1145/ 2024724.2024737

[11] X. Chen, J. Zheng, Y. Chen, H. Li, W. Zhang, S. W. Liao and J. Wang: ASP- 
DAC (2012) 807. DOI:10.1109/ASPDAC.2012.6165066

[12] M. Jadhav, Y. Dandawate and N. Pisharoty: Int. J. Eng. Technol. 5 (2013) 1555.

[13] H. R. Sheikh, M. F. Sabir and A. C. Bovik: IEEE Trans. Image Process. 15 (2006) 3440. DOI:10.1109/TIP.2006.881959

[14] Z. Wang, A. C. Bovik, H. R. Sheikh and E. P. Simoncelli: IEEE Trans. Image Process. 13 (2004) 600. DOI:10.1109/TIP.2003.819861

[15] M. Dong, Y.-S. Choi and L. Zhong: DAC (2009) 652. DOI:10.1145/1629911. 1630084

[16] CHI MEI: C0240QGLA-T Approval Product Specification (2008) http://html. alldatasheet.com/html-pdf/317814/AZDISPLAYS/C0240QGLA-T/604/1/ C0240QGLA-T.html, accessed October 2014.

[17] Texas Instruments: TPS65138 Dual Output AMOLED Display Power Supply Datasheet (2013) http://www.ti.com/lit/ds/symlink/tps65138.pdf.

\section{Introduction}

Energy consumption is an important issue in mobile embedded systems because they are battery-driven. Display is a major power consuming component in mobile devices. To reduce the power consumption of displays, a lot of techniques have been researched. Till now, liquid crystal display (LCD) is the most widely used display technology in mobile devices. The power consumption of LCD is almost constant because most of the power is consumed by its backlight unit (BLU). Through backlight dimming techniques, power consumption of LCD can be reduced significantly $[1,2]$.

Active matrix organic light-emitting diode (AMOLED) display is replacing LCD as a new generation display technology due to flexibility, brilliant colors, wide viewing angle, and fast response compared with conventional LCD [3, 4]. Unlike LCD, AMOLED display consumes different power when it shows different colors due to its self-illuminating characteristic. Thus, its power consumption depends on the displayed contents. To reduce the power consumption of AMOLED display, different techniques are needed because BLUs are not necessary. In prior work, low-power techniques for AMOLED displays are researched using two different ways. One is color transformation and the other is dynamic voltage scaling (DVS) of a display pannel.

The color transformation technique changes the colors of the pixels in order to save their power consumption. Recently, many color transformation techniques have been studied actively. Greenvis [5] is one of the techniques which is used for a data visualization purpose. Chameleon [6] targets mobile web browsers by transforming the colors of web pages. Other techniques like FOCUS [7] and Anand's [8] only focus on some limited region of the display. PARVAI [9] is a grid-based color transformation technique which focuses on reducing the blue colors of the pixels and targets game applications. In prior work, only the usability of the display content is considered instead of the fidelity. In addition, all the previous techniques focus on specific applications. Our work focuses on fidelity rather than usability and targets generic image displaying applications. 
DVS is a technique which changes the supply voltage of a display pannel in order to save its power consumption depending on the display content. Shin et al. [10] made the fist research for OLED but their work is applied to PMOLED displays which is outdated. An improved technique called fine-grained dynamic voltage scaling (FDVS) [11] partitions an AMOLED display pannel into multiple areas and applies DVS to each area depending on its content. This work employs simulations only and is not implemented on a real platform. Thus, research on real mobile AMOLED display is required to show their real power consumption and image qualities.

In the meantime, when DVS is applied, some pixels can be distorted since lower voltage is supplied. To recover the distortion, pixel compensation is conducted by increasing the pixel values to obtain the original luminance of the pixels [11]. Pixel compensation is considered as a subset of color transformation. Prior DVS work mainly depends on pixel compensation rather than color transformation to recover the lost luminance caused by voltage scaling.

In perceptually uniform color spaces like the CIELab color space, a small color change is tolerantly allowed to the human visual system (HVS) [12]. Thus, in the CIELab color space, transforming the pixel colors of an image uniformly into lower power-consuming colors is expected to guarantee HVS-awareness as well as high power saving for AMOLED displays. Our work tackles utilizing color transformation as well as pixel compensation so that DVS may achieve more power saving and better visual satisfaction.

In this paper, a novel approach of combing color transformation and DVS is proposed in the aspect of both power saving and visual quality. By combining color transformation and DVS synergistically, high power saving and good visual quality can be achieved. For evaluation of the proposed approach, a linux-based embedded board which is interfaced with a commercial mobile AMOLED display module is developed. To the best of our knowledge, this is the first work which applies DVS on a commercial mobile AMOLED display module.

\section{CTDVS: Color transformation-based DVS}

\subsection{Design of CTDVS}

Our new approach is called color transformation-based DVS (CTDVS). In this section, we will explain how the low-power color transformation technique and DVS are combined to achieve both high power saving and HVS-awareness fully.

The Euclidean distance in the CIELab color space represents human perceptual difference. Thus, we have chosen this color space for low-power color transformation. In the color space, we search for a vector which satisfies both low-power consumption and HVS-aware visual quality, and all the pixels of an image will be moved concurrently toward its direction if a vector is found. Eq. (1) shows the Euclidean distance in the CIELab space and the $h$ value represents the distance between the original color ( $L, a, b)$ and transformed color $\left(L^{\prime}, a^{\prime}, b^{\prime}\right)$ in this space. The amount of power saving and the visual quality can be controlled by the $h$ value. The bigger the distance, the more different the two colors are. If $h$ is too big, more power can be saved but the visual quality of the image will be affected negatively. 
Thus, $h$ having a good tradeoff between the power saving and visual quality should be selected.

$$
\left(L^{\prime}-L\right)^{2}+\left(a^{\prime}-a\right)^{2}+\left(b^{\prime}-b\right)^{2}=h^{2}
$$

In DVS, we can save additional power by selecting a lower voltage than a required one while the luminance of some pixels having high brightness is sacrificed. The reason is that most of the pixels have less values than the maximum pixel value. As shown in Fig. 1, every voltage has a different maximum luminance value. As a result, pixel compensation can only be applied within the limit of these maximum luminance values. Some pixels whose luminance values are above the maximum luminance value, which can be produced by the selected voltage, are saturated with the maximum luminance value. The number of saturated pixels can be varied depending on the required power saving and visual quality of the transformed image. The percentage of the number of pixels, which are not saturated, is called "saturation threshold". The amount of power saving and visual quality can be controlled by saturation threshold. Thus, saturation threshold having a good tradeoff between the power saving and visual quality should be selected as the $h$ value does.

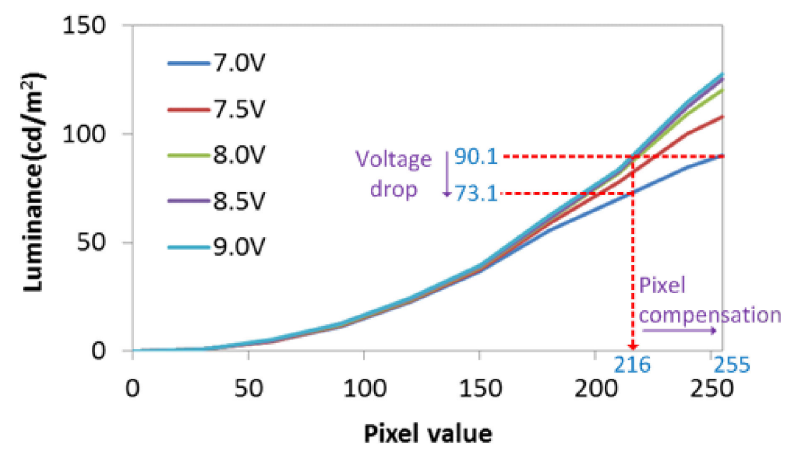

Fig. 1. Pixel compensation of the green channel when Vdd is dropped from $9 \mathrm{~V}$ to $7 \mathrm{~V}$.

After color transformation, DVS will be applied with a selected saturation threshold value depending on the $h$ value. Searching pairs of $h$ and saturation threshold simultaneously in terms of maximum power saving and high visual quality will result in an optimal pair. In addition, because every images has different characteristics, all possible pairs of $h$ and saturation threshold should be traversed, while taking image characteristic into consideration. Now we can combine lowpower color transformation and DVS smartly in order to accomplish both lowpower consumption and HVS-awareness.

\subsection{Algorithm of CTDVS}

This section describes the overall algorithm of CTDVS. It has two main parts: offline and online. In the offline algorithm, image categories are established based on the average luminance of reference images to patternize an input image. An optimal pair of $h$ and saturation threshold is assigned for each category. The online algorithm identifies the category of an input image and uses its corresponding pair of $h$ and saturation threshold to transform the image. 
In the offline algorithm, four categories with different average luminance ranges are built. The first category is for dark images with very low average luminance values and the last (fourth) category is for bright images with very high average luminance values. The reference images in the LIVE [13] database are used for categorization. In each category, the darkest image is chosen. When CTDVS is applied, the darkest image generally will have the least SSIM (Structural Similarity) index values.

SSIM is a HVS-aware image quality mertic combining luminance, contrast, and structure assessment of a modified image over an original one [14], and it approaches 0 as two images become quite different and gets close to 1 as they become identical. SSIM has been widely used to evaluate the image quality and thus is adopted in this work although it is not optimal in the aspect of the HVS. The research on an optimal HVS-aware image quality metric is beyond the scope of this paper.

If the darkest image in a category satisfies the required SSIM, all the other images in the same category and any input images which will be categorized into the same category can also satisfy the required SSIM. Hence, the darkest image in a category is selected as a representative one to guarantee HVS-awareness conservatively.

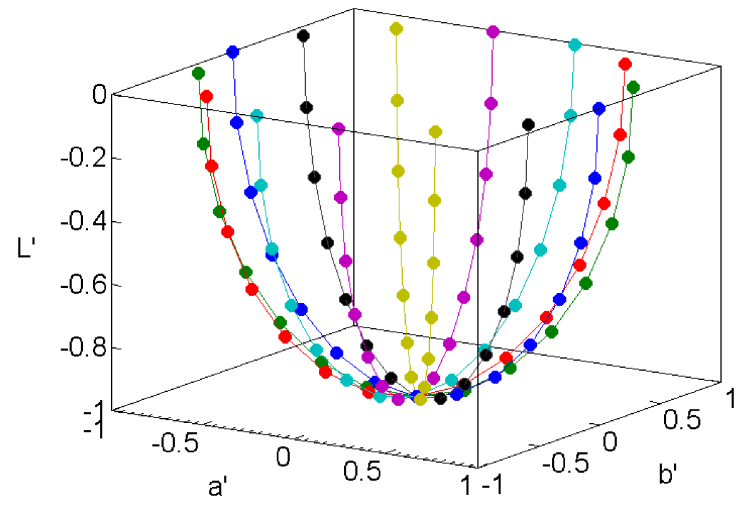

Fig. 2. 106 unit vectors on the lower hemisphere in the CIELab color space.

First of all, in the offline algorithm, an optimal direction (or vector) in the CIELab color space is identified. This direction will have optimal power saving and high HVS-awareness when the pixels of an image are changed toward it concurrently. To identify it, candidate vectors in the CIELab color space are searched with their power consumption and visual quality compared. As is shown in Fig. 2, 106 uniformly distributed points on the surface of the lower hemisphere are used as the candidate vectors. When $h$ equals 1 in Eq. (1), candidate vectors become unit ones. Thus, if $h$ varies, scaled 106 unit vectors will be used as candidate vectors.

All the pixels of an input image are transformed concurrently by each vector to generate different transformed images. The power consumption and the visual quality of each of the transformed images are compared. In our experiments, we repeated such a search with 20 input images, identified which vector has optimal 
balance of power saving and HVS-awareness of the resulting images for most of input images, and selected it as the optimal vector.

In order to compare the visual quality of the resulting images, a user study with 10 subjects were conducted. HVS-awareness was assessed with their naked eyes. And for power comparison, a pixel-level power model of an AMOLED display is used, which is given in Eq. (2). In Eq. (2), $f, k$, and $m$ are the power consumption of $\mathrm{R}, \mathrm{G}$, and B sub-pixels, respectively, and it is assumed that each function is linear with each sub-pixel. $P_{\text {pixel }}$ is the pixel-level power consumption. The total power consumption of an image is found by adding the power consumption of all the pixels in the image [15].

$$
P_{\text {pixel }}(R, G, B)=f(R)+k(G)+m(B)
$$

The vectors on the upper hemisphere will make the original pixel have an increase luminance value, causing more power consumption. Thus, the search for the optimal vector is conducted only for candidate vectors on the lower hemisphere. After extensive search, the optimal vector is found at the bottom end of the lower hemisphere.

Fig. 3(a) shows the offline algorithm. In line 4, the low-power color transformation function addressed in Fig. 3(c) is called. In this function, the pixel colors of an image are converted from the RGB color space to the CIELab color space. After that, each pixel color is transformed from an original CIELab color (L, a, b) to a HVS-aware low-power color ( $\left.\mathrm{L}^{\prime}, \mathrm{a}^{\prime}, \mathrm{b}^{\prime}\right)$ using the optimal direction with a given $h$. Then, each pixel color is converted back to the RGB color space.

In lines 5-7, DVS is applied according to a selected saturation threshold. The saturation threshold value in a unit of percentage is used to decide the maximum pixel value (sth_val) which makes the fraction of unsaturated pixels over all pixels equal to saturation threshold for each RGB channel. To calculate each sth_val, a histogram per channel is built. For example, in the histogram of Fig. 4, saturation threshold is 90 and the sth_val at this saturation threshold are found to be 164 for the green channel.

Then, the supply voltage for the AMOLED pannel is scaled to the selected voltage and pixel compensation is conducted to recover the lost luminance by increasing pixel values. As mentioned in subsection 2.1, pixel compensation can only be applied within the limit of the maximum luminance value of a selected supply voltage. In Fig. 1, when the supply voltage is scaled down from $9 \mathrm{~V}$ to $7 \mathrm{~V}$, the luminance at the pixel value of 216 decreases from 90.1 to 73.1 . To compensate the lost luminance, the pixel value of 216 can be increased to 255 , at which the maximum luminance of 90.1 is obtained for $7 \mathrm{~V}$. The pixel values below 216 will be compensated similarly but the pixel values above 216 will be saturated to 255 after compensation. All pairs of such a pixel value for compensation and its corresponding supply voltage value will be stored in a Voltage Table except 9.0 V, which is shown in Fig. 4. And, in our work, prebuilt compensation LUTs are used instead of calculating pixel values for compensation at run-time for performance optimization.

Fig. 4 shows how sth_val and the Voltage Table are used to select a proper voltage. The calculated sth_val from the histogram will be compared against pixel 


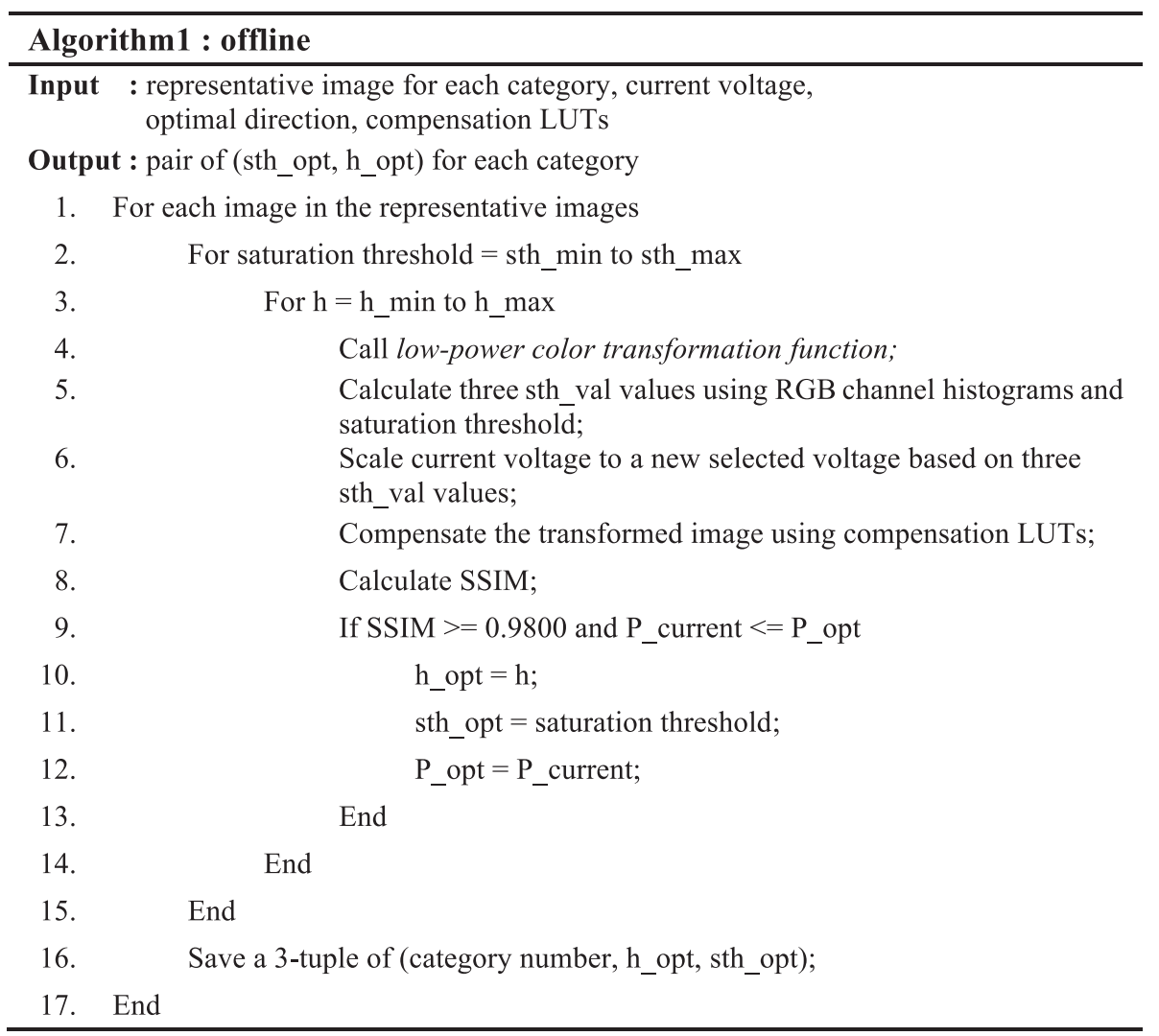

(a)
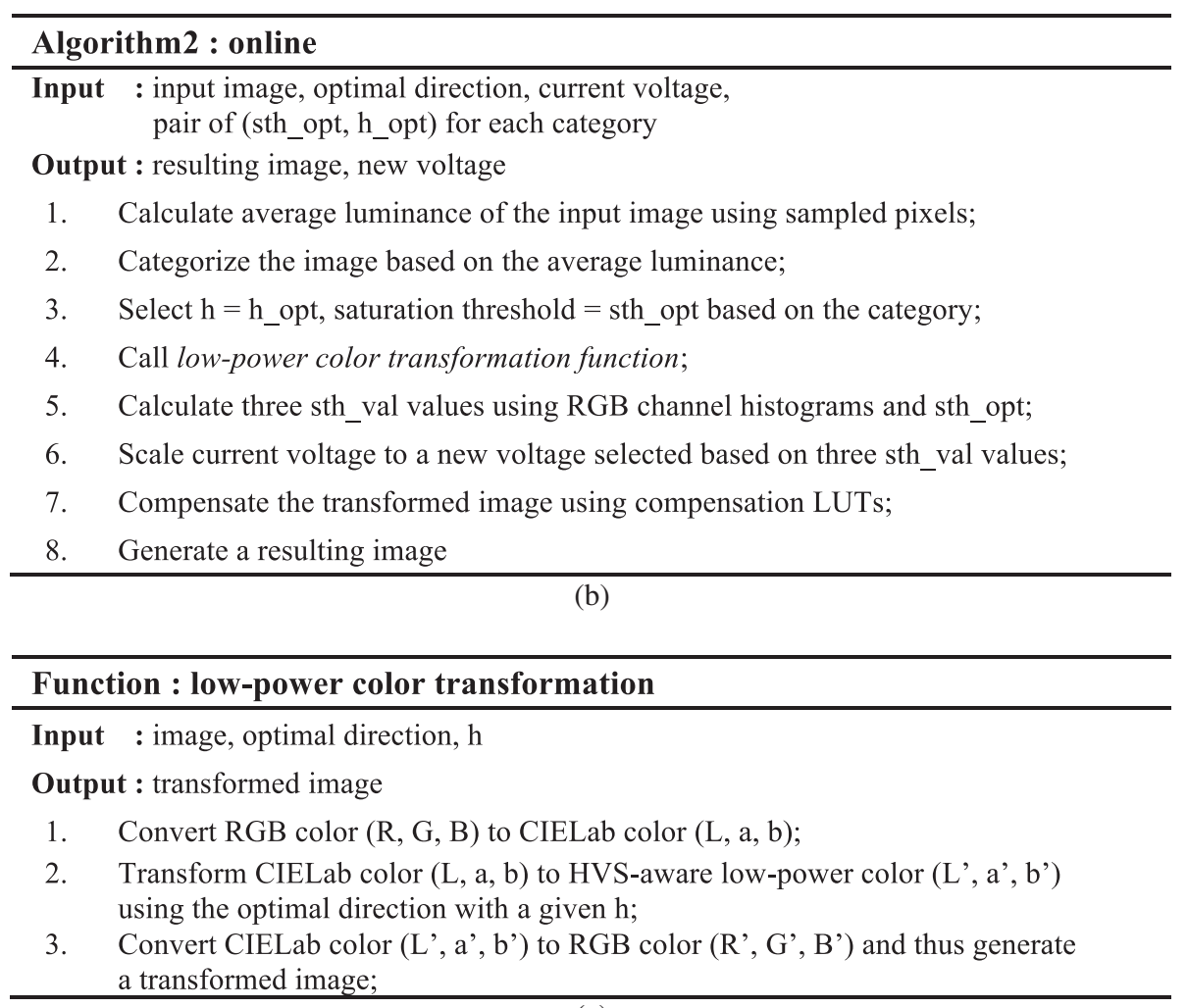

(c)

Fig. 3. Pseudo code of CTDVS: (a) offline and (b) online algorithms. (c) low-power color transformation function. 


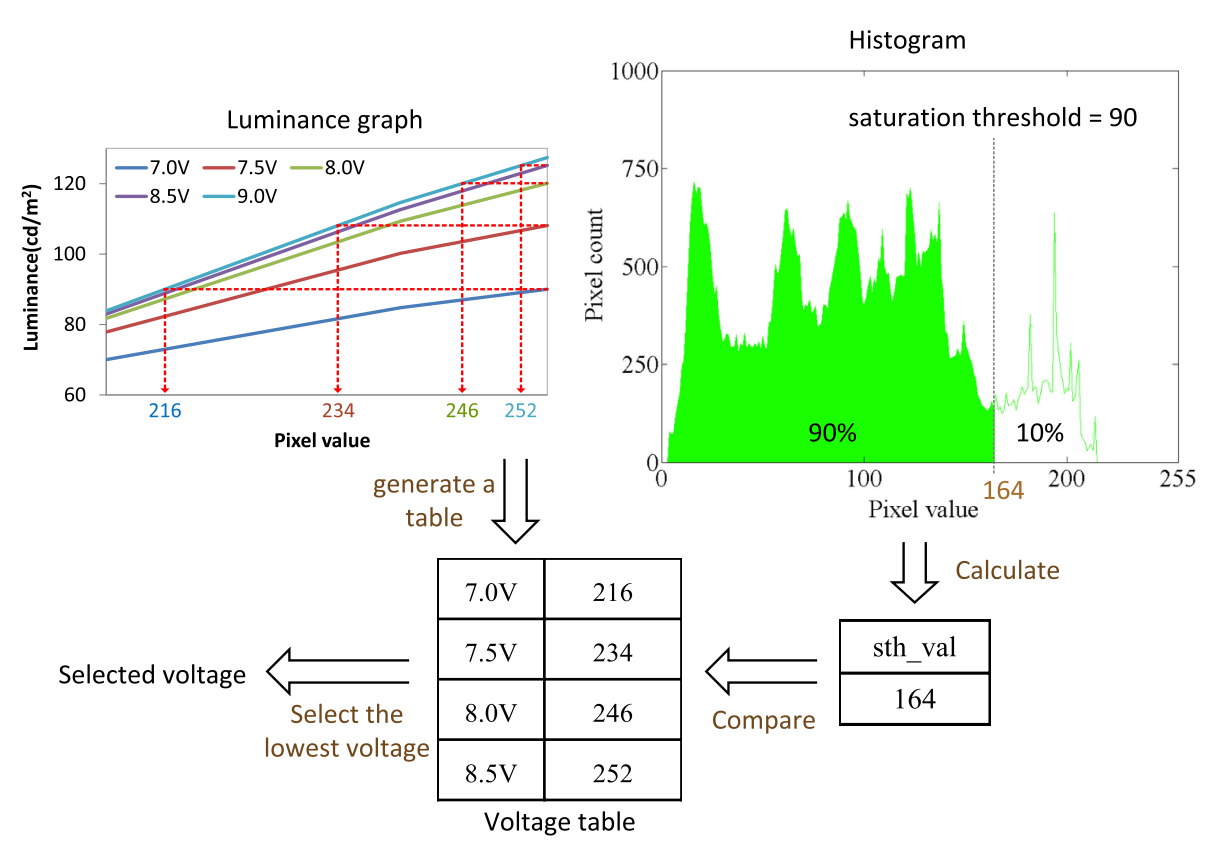

Fig. 4. Overall flow of voltage selection in DVS

values in the Voltage Table and then the lowest among all the voltage levels whose pixel values are greater than or equal to sth_val will be selected. For example, in Fig. 4, sth_val is 164 and the lowest supply voltage which can meet this value is $7.0 \mathrm{~V}$. After aquiring the three lowest voltages for RGB channels, finally, the biggest voltage is selected among them. This is a conservative way to make pixels of all channels compensated perfectly.

In lines 8-13, SSIM and power consumption of the resulting image are compared with those of the original image. 1, 2.3, 3, and 4 are used for the $h$ value with the minimum $h\lrcorner \min =1$ and the maximum $h \_\max =4$. And 85, 90, 95, and 100 are used for saturation threshold with the minimum sth_min $=85$ and the maximum sth_max $=100$. When $h$ and saturation threshold vary, among all the pairs whose SSIM value is greater than or equal to 0.9800, an optimal pair ( $h \_o p t$, sth_opt) which consumes the lowest power (P_opt) is selected. The SSIM value between 0.9800 to 1.000 indicates that the modified image has high visual quality [2]. The power consumption is measured using the experimental setup described in Section 3. In line 16, the found pair (h_opt, sth_opt) is saved with the category number finally.

The online algorithm is shown in Fig. 3(b). In lines 1-2, sampled pixels of an input image are used to get its average luminance in order to reduce computational overheads. Based on the average luminance, an image category is identified to take the characteristic of the input image into consideration. In line 3, according to the identified category, a corresponding optimal pair (h_opt, sth_opt) which is found in the offline algorithm is used to achieve optimal power saving and HVS-awareness. In line 4, the low-power color transformation function is invoked. In lines 5-7, using the optimal saturation threshold (sth_opt), sth_val are calculated for each RGB channel. After that, the lowest possible supply voltage is selected based on the sth_val values as shown in Fig. 4. Finally, the transformed image is compensated using the compensation LUT. 


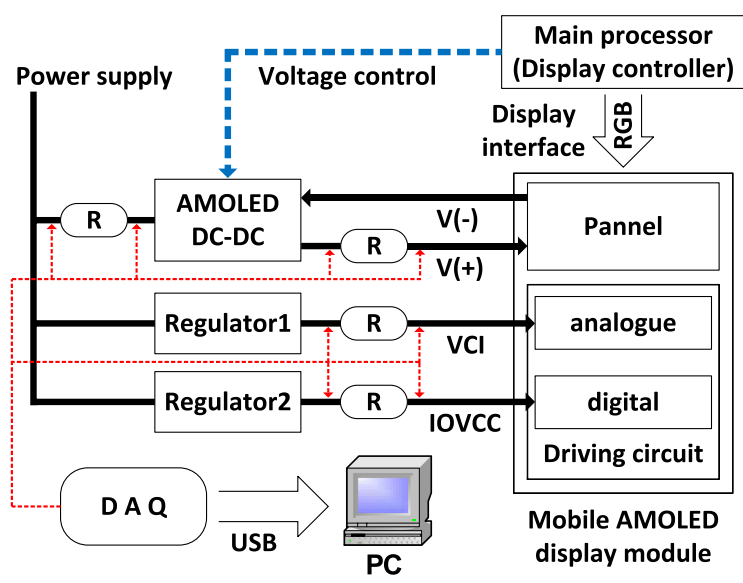

Fig. 5. Block diagram of the AMOLED display system with an implementation of CTDVS

\section{Experiments}

In order to implement CTDVS, a linux-based embedded board which is interfaced with a commercial mobile AMOLED display module was developed. The board enables performing DVS and measuring the power consumption of the display accurately. In Fig. 5, the block diagram of the AMOLED display system with an implementation of CTDVS is shown. The AMOLED display module has three different voltage sources. One of them is a programmable AMOLED DC-DC converter which supplies power to the AMOLED pannel. The other sources are two voltage regulators which supply power to the analog and digital circuits of the display module. By programming the DC-DC converter, the supply voltage of the pannel can be controlled.

To measure voltage and current that supply power to the mobile AMOLED display module, current sensing resistors are connected to individual outputs of voltage sources. Another current sensing resistor is connected to the input of the AMOLED DC-DC converter to measure its own power consumption. A data acquisition (DAQ) board is used to measure the power consumption of the current sensing resistors. The data from the DAQ is transmitted to a PC and the power consumption is analyzed.

The experimental environment is shown in Fig. 6. In Fig. 6(a), our luminance and power measurement setup is shown. Using this setup, the relationship among the supply voltage, pixel value, and luminance is established for the RGB channels in the RGB color space and the power consumption of the AMOLED display module is measured. To measure the luminance of the AMOLED panel, a Konica Minolta LS-110 luminance meter is used. Fig. 6(b) shows the CTDVS-implemented board and its connection with DAQ. The target AMOLED display module is C0240QGLA-T from CHI MEI which has a 2.4" display panel and a resolution of $240 \times 320$ [16]. The AMOLED DC-DC converter IC TPS65138 from TI [17] is used for DVS and NI USB-6210 DAQ is used for power measurement.

Experiments are conducted using the reference images from the open LIVE database and two classic images Lenna and Baboon. The database images are used for categorization in the offline algorithm. Fig. 7 shows the results of CTDVS 


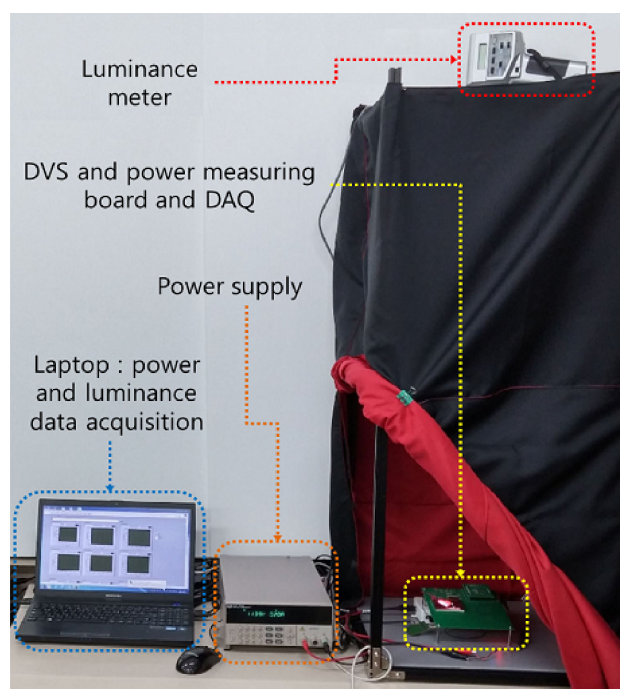

(a)

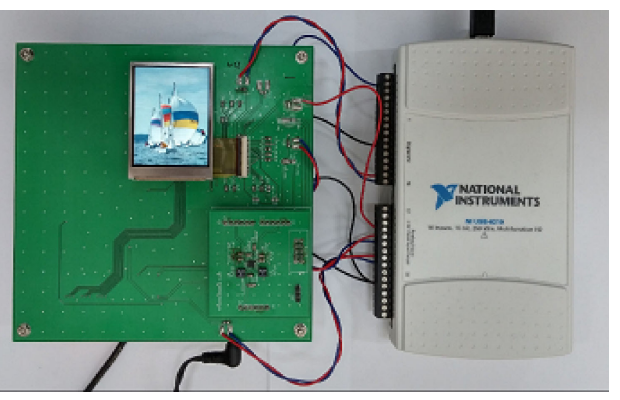

(b)

Fig. 6. Experimental environment. (a) Experimental setup for luminance and power measurement of an AMOLED display module (b) CTDVS board and DAQ.

applied to a test image from the LIVE database when saturation threshold and $h$ vary. Fig. 7(a), (b), and (c) show the resulting images captured by a digital camera when saturation thresholds are 100, 95, and 90, respectively with a fixed value of $h=0$. It is found that the change of saturation threshold does not have a noticeable effect on the HVS-aware perceptuality. Table I shows the supply voltages, SSIM values, and power savings for the images (a), (b), (c), and (d) in Fig. 7. As shown in the table, when saturation threshold decreases, the image quality (i.e., SSIM) also decreases while the percentage of power saving increases. The reason is that a decrease in saturation threshold leads to a decrease in the sth_val which will cause a lower supply voltage selection and also leads to increase the number of saturated pixels which can have negative effects on the image quality. When saturation threshold is 90 and 85 , the percentage of power saving is almost same because the lowest supply voltage $(7 \mathrm{~V})$ is selected for both cases.

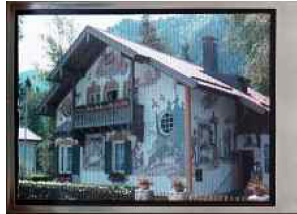

(a) $h=0, s t h=100$

(original)

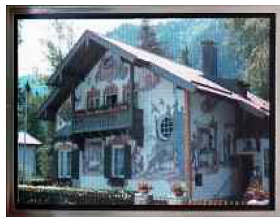

(e) $h=1, s t h=100$

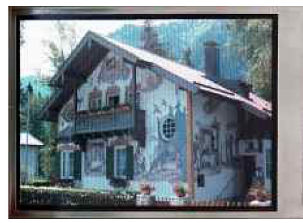

(b) $h=0, s t h=95$

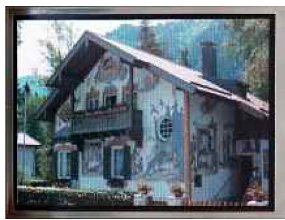

(f) $h=2.3, s t h=100$

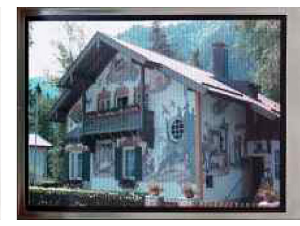

(c) $h=0, s t h=90$

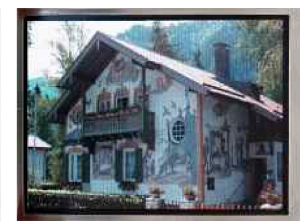

(g) $h=3, s t h=100$

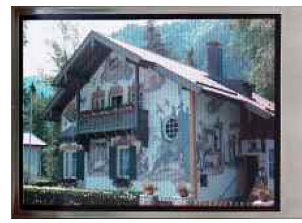

(d) $h=0, s t h=85$

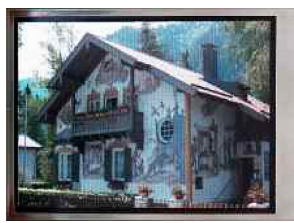

(h) $h=4, s t h=100$

Fig. 7. Results of CTDVS on a test image when $h$ and saturation threshold (sth) vary. 
Table I. Comparison of Vdd, SSIM, and power saving for Fig. 7(a)-(d).

\begin{tabular}{|c|c|c|c|}
\hline Saturation threshold & Vdd (V) & SSIM & Power saving (\%) \\
\hline 100 & 9 & 1 & - \\
\hline 95 & 8 & 0.9892 & 11.8 \\
\hline 90 & 7 & 0.9842 & 24.0 \\
\hline 85 & 7 & 0.9842 & 24.1 \\
\hline
\end{tabular}

Table II. Comparison of Vdd, SSIM, and power saving for Fig. 7(e)-(h).

\begin{tabular}{|c|c|c|c|}
\hline $\mathrm{h}$ & $\mathrm{Vdd}(\mathrm{V})$ & SSIM & Power saving (\%) \\
\hline 1 & 8.5 & 0.9888 & 7.0 \\
\hline 2.3 & 8.5 & 0.9863 & 10.1 \\
\hline 3 & 8 & 0.9845 & 18.6 \\
\hline 4 & 8 & 0.9807 & 19.0 \\
\hline
\end{tabular}

Fig. 7(e), (f), (g), and (h) are the resulting images when the $h$ values are 1, 2.3, 3 , and 4 , respectively with a fixed value of saturation threshold $=100$. Table II shows the supply voltages, SSIM values, and power savings for the images (e), (f), $(\mathrm{g})$, and (h) in Fig. 7. In the table, when $h$ increases, the SSIM value decreases but the percentage of power saving increases. This is because an increase in $h$ reduces the brightness of an image since the luminance in the CIE Lab space decreases and thus the image quality is degraded while the power saving increases. As an image is transformed to a darker image in our low-power color transformation, additional power saving can be achieved because a lower supply voltage can be selected due to the decrease in all of the pixel values.

Fig. 8 shows the results of CTDVS applied to the Lenna and Baboon images. These resulting images are obtained after the CTDVS algorithm applied fully. Thus, the optimal $h$ and saturation threshold for each input image are selected automatically at runtime. And Table III shows the corresponding supply voltages, $h$ values, saturation threshold values, power consumption, and power savings for two original images and their resulting ones. As shown in the table, 29.5\% and $29.8 \%$ of power savings are achieved for the Lenna and Baboon images, respec-

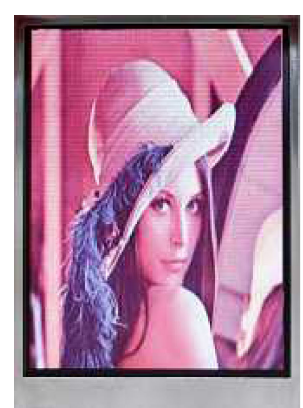

(a)

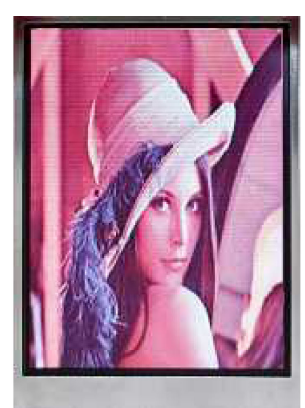

(b)

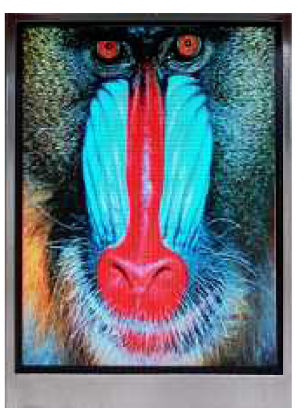

(c)

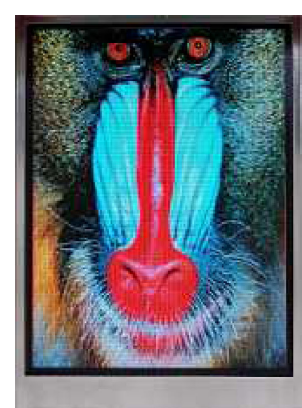

(d)

Fig. 8. Original and CTDVS-applied images. (a) Lenna original (b) Lenna applied (c) Baboon original (d) Baboon applied. 
Table III. Comparison of original and CTDVS-applied images.

\begin{tabular}{|c|c|c|c|c|}
\hline & \multicolumn{2}{|c|}{ Lenna } & \multicolumn{2}{c|}{ Baboon } \\
\hline & Original & CTDVS & Original & CTDVS \\
\hline Vdd (V) & 9 & 7 & 9 & 7 \\
\hline $\mathrm{h}$ & - & 3 & - & 4 \\
\hline Saturation threshold & - & 85 & - & 85 \\
\hline SSIM & 1 & 0.9818 & 1 & 0.9856 \\
\hline Measured power (mW) & 323.2 & 227.8 & 261.3 & 183.4 \\
\hline Power saving (\%) & - & 29.5 & - & 29.8 \\
\hline
\end{tabular}

tively, and the SSIM values for both resulting images are greater than 0.9800 . The lowest supply voltage $(7 \mathrm{~V})$ is selected and saturation threshold for both images is 85 . The values of $h=3$ and $h=4$ are selected in the online algorithm and used in the low-power color transformation for the Lenna and Baboon images, respectively. The results indicate that CTDVS achieves high power saving while guaranteeing HVS-awareness in the aspect of perceptual satisfaction.

\section{Conclusion}

In this paper, we proposed a novel approach of low-power color transformationbased DVS called CTDVS for the purpose of optimal power saving and HVSaware visual satisfaction on mobile AMOLED displays. It dynamically adjusts allowable perceptuality and saturation threshold based on the luminance value of a given image and transforms the colors of the image pixels while satisfying human visuality. Then, a smart DVS technique is performed according to the selected perceptuality and saturation threshold at run-time. CTDVS was implemented on a mobile AMOLED display board with a well-devised programmable voltage controller. Experimental results show that CTDVS can save on average $29.6 \%$ of the display power with high satisfaction to the HVS over two classic images. As a future work, we plan to make research on implementing CTDVS for video play.

\section{Acknowledgments}

This work was supported by the Ajou university research fund. 